\title{
Investigating nanoparticle surface energetics using 4D-STEM
}

\author{
B.D. Esser ${ }^{1,2}$, A.M. Funston ${ }^{3}$, J. Etheridge ${ }^{1,2}$ \\ ${ }^{1}$ Monash Centre for Electron Microscopy, Monash University, VIC, 3800, Australia \\ ${ }^{2}$ Department of Materials Engineering, Monash University, VIC, 3800, Australia \\ ${ }^{3}$ School of Chemistry, Monash University, VIC, 3800, Australia \\ Bryan.Esser@monash.edu
}

Metallic nanoparticles have been shown to have a wide variety of applications from catalysis to plasmonics and medicinal drug delivery [1-3]. The onset of functional properties not seen in bulk can be attributed to and finely controlled by particle size, shape, homogeneity, and chemistry [4-6]. Given nanoparticles' small size - typically sub-100 nm - their properties can be strongly influenced by crystallographic defects such as twinning, interfaces, and surfaces. As such, a fundamental study of the energetics of atomic mobility at the surface of a nanoparticle can provide an invaluable understanding of the relationship between crystal structure and functional properties. Moreover, surface energetics play a key role in controlling nanocrystal growth and shape. In this study, Au nanoparticles with different organic ligands, and therefore different surface stabilities, are investigated in real space using quantitative atomic resolution aberration corrected scanning transmission electron microscopy (STEM).

STEM imaging has become one of the leading methods of materials characterization, and aberration correction has made atomic resolution imaging widely available. Advanced imaging and image processing techniques have been developed to quantify specimen features including thickness projected along the path of the beam [7-12]. Such quantitative methods generally rely on high angle annular dark field (HAADF) STEM, whereby images are formed using electrons that have been scattered to high angle with a strong atomic number dependence (so-called $Z$ contrast). While these techniques have been applied to great effect under specific controlled conditions, most of the beam-specimen interaction is discarded both from lower scattering angles and via the angular and azimuthal integration of HAADF detectors. Such information is rich with detail about specimen morphology and can be used to improve quantitative precision when paired with image simulation.

Recently, the development of fast, high-dynamic range, direct electron detectors has made it possible to record the scattering distribution $\left(\mathrm{k}_{\mathrm{x}}, \mathrm{k}_{\mathrm{y}}\right)$ as a function of probe position $\left(\mathrm{r}_{\mathrm{x}}, \mathrm{r}_{\mathrm{y}}\right)$, generally referred to as 4D-STEM [13-15]. Such detectors have noise levels well below that of single-electron strikes, making them ideal for quantitative imaging. By collecting the whole scattering distribution at each probe position rather than integrating over an annulus, images can be formed using specific regions of the diffraction pattern that are most strongly affected by variations in thickness. Additionally, several images can be formed from the same dataset using different scattering regimes to further constrain thickness measurements.

Here we report on advances in quantitative thickness determination using 4D-STEM paired with multislice simulations. A detailed comparison of the advantages and challenges of using 4D-STEM opposed to conventional HAADF-STEM will be covered. Furthermore, we apply these 4D-STEM atom counting techniques to metallic nanoparticle systems to probe the energetics of beam-induced surface atom motion. The implications of such surface energetics on nanoparticle properties will be discussed.

[1] Dreaden, et al. (2012). Chem. Soc. Rev. 41, 2740-2279.

[2] Sau, et al. (2010). Adv. Mater. 22, 1805-1825.

[3] Zhou, et al. (2011). Chem. Soc. Rev. 40, 4167-4185.

[4] Balbuena, P. and Seminario, J. (2006). Nanomaterials: Design and Simulation, Elsevier: Amsterdam.

[5] Yin, Y. and Alivisatos, A.P. (2005). Nature 437, 664-670.

[6] Henry (2005). Prog. Surf. Sci. 80, 92-116.

[7] LeBeau, et al. (2010). Nano Lett. 10, 4405-4408.

[8] Van Aert, et al. (2013). Phys. Rev. B 87, 064107.

[9] De Backer, et al. (2013). Ultramicroscopy 134, 23-33.

[10] Katz-Boon, et al. (2013). Ultramicroscopy 124, 61-70.

[11] Katz-Boon, et al. (2011). Nano Lett. 11, 273-278.

[12] Dwyer, et al. (2012). Appl. Phys. Lett. 100, 191915.

[13] Tate, et al. (2016). Microsc. Microanal. 22, 237-249.

[14] Ballabriga, et al. (2011). Nucl. Instrum. Methods Phys. Res. 633, S15-S18.

[15] Johnson, et al. (2018). Microsc. Microanal. 24, 166-167.

\section{Keywords: 4D-STEM; nanoparticle; quantitative STEM;}

The authors acknowledge the use of the instruments and scientific and technical assistance at the Monash Centre for Electron Microscopy, a Node of Microscopy Australia. This research used equipment funded by Australian Research Council grant LE0454166. 